\title{
Gonadal hormones and cognitive performance
}

\author{
PAULINE KOMNENICH and DAVID M. LANE \\ Tulane University, New Orleans, Louisiana 70118 \\ and \\ RICHARD P. DICKEY and SERGIO C. STONE \\ Department of Obstetrics and Gynecology, Louisiana State University Medical Center \\ New Orleans, Louisiana 70112
}

\begin{abstract}
The relation of gonadal hormones to cognitive performance was studied in females with ovulatory menstrual cycles, females on oral contraceptives, and males. For the females with the ovulatory menstrual cycles, the highest performance on simple repetitive tasks and the lowest performance on tasks requiring inhibition occurred in the ovulatory phase. Serum estradiol was found to be related to test performance.
\end{abstract}

In the past several years there has been a series of articles devoted to the question of the relationship between cognitive performance and physical maturity (Broverman, Broverman, Vogel, Palmer, \& Klaiber, 1964; Broverman \& Klaiber, 1969; Petersen, 1976; Waber, 1976). These studies have found that within a given age level, physically mature male adolescents perform better on speeded tasks such as naming colors or cancelling numbers than one would expect on the basis of the overall level of performance. In contrast, the best performance of the less mature male adolescents occurs on tasks such as the Embedded Figures Test (EFT) and the Block Design Test (BDT). Broverman et al. (1964) suggested that the tasks differentiating these two groups fall into two categories: (1) those requiring simple repetitive responses and (2) those which require the inhibition of attention to salient stimulus attributes or the inhibition of overlearned responses. Physically mature subjects, then, would be said to excel on the former type of task and less mature subjects on the latter.

While the evidence for a relationship between physical maturity and cognitive performance has been quite consistent, the basis of the relationship is less clear. Broverman et al. (1964) proposed that the relationship is mediated by gonadal hormones. According to this argument, sex hormones, by inhib-

We thank Dr. A. Gerall and Dr. I. Bilodeau, Department of Psychology, Tulane University, New Orleans, for their sponsorship, contributions, and helpful discussions; and M. Pratt and J. Stagg for their technical assistance. This work was supported through a postdoctoral fellowship from NIH Child Health and Human Development awarded to P. Komnenich. Requests for reprints should be addressed to the first author, School of Nursing and Allied Health Professions, Saint Louis University, St. Louis, Missouri 63104. iting the enzyme monoamineoxidase (MAO), raise the level of central norepinephrine and are therefore associated with activation or arousal. Activation is hypothesized to facilitate performance on simple repetitive tasks and to impede performance on inhibition tasks.

More recent data are generally consistent with this view, although the evidence is still far from conclusive. Zolovick, Pearse, Boehlke, and Eleftheriou (1966) found a relationship between the phase of the menstrual cycle and MAO activity at the level of the hypothalamus in rats. Briggs and Briggs (1972) and Klaiber, Kobayashi, Broverman, and Hall (1971) found that plasma MAO activity varied systematically over the course of the menstrual cycle in women, the lowest levels of activity occurring when estradiol levels were highest. Redmond, Murphy, Baulu, Ziegler, and Lake (1975) investigated the relationship of platelet MAO and estradiol. High levels of platelet MAO were associated with high levels of estradiol. It was suggested that since platelet life span is about 10 days, the high MAO activity might have resulted from several days of low estrogen 10 or more days earlier. This study by Redmond et al. (1975) failed to replicate the earlier findings of a relationship between plasma MAO and estradiol, although there was a trend in that direction. In a study of MAO in human brain tissue obtained at autopsy, Robinson, Sourkes, Nies, Harris, Spector, Bartlett, and Kaye (1977) found significant correlations of MAO activity with sex and age, suggesting that endocrine factors contribute to the between-individual variance in MAO. Norepinephrine is believed to be metabolized by at least one form of MAO (Yang \& Neff, 1974). Broverman's theory received considerable indirect support from both animal and human research (Broverman, Klaiber, Kobayashi, \& Vogel, 1968), yet 
it suffers from the fact that hormonal levels have never been measured directly. They have been inferred from physical characteristics such as the amount of body hair present.

Because estrogen and testosterone are hypothesized to have similar activating effects (Broverman et al., 1968), one might expect that the relationship between maturity and cognitive performance found with males would also hold for females. In fact, Waber (1976) did find the expected relationship for both males and females. Although Waber used many of the same tasks as did Broverman et al. (1964), she did not accepi Broverman's categorization of these tasks into those requiring simple repetitive responses and those requiring inhibition. Instead, she referred to these two sets of tasks as tests of verbal and spatial ability, respectively. The EFT and the BDT have often been used to assess the ability of subjects to inhibit attention to salient but irrelevant cues (Case \& Globerson, 1974; Pascual-Leone, 1970); however, there is good reason to believe that these tests share considerable variance with spatial ability (Sherman, 1967; Smith, 1964). The use of the speed of naming colors test and the Digit Symbol Test (DST) as measures of verbal ability, however, is questionable. When Waber performed a reanalysis of her data (Waber, 1977), she found support for her contention that the EFT and the BDT can be thought of as measures of spatial ability but no support for the idea that the speed of naming colors test or the DST reflect verbal ability. A factor analysis revealed three factors: (1) a spatial ability factor defined by the EFT, the BDT, and a traditional test of spatial ability, (2) a factor with high loadings on only the DST and the speed of naming colors test, and (3) a factor consisting of only a test of verbal fluency.

In the present study, we tested predictions derived from the theory of Broverman and his colleqgues. According to this theory, one would expect that performance on simple repetitive tasks relative to inhibition tasks would vary as a function of a woman's menstrual cycle. Using the term "automatization"' to refer to the degree to which one performs better on simple repetitive tasks than on inhibition tasks (Broverman et al., 1964), one would expect women to be most automatized during the estrogen surge which occurs just prior to ovulation. For male subjects, it was predicted that automatization would be positively correlated with testosterone level. Because of the controversy over whether the EFT is best characterized as measuring inhibition or spatial ability, we used not only the EFT but also a task requiring subjects to count backward. The advantage of this task is that although it certainly is not a spatial test, it has been hypothesized by Broverman et al. (1968) to require inhibition of the overlearned tendency to count forward.

\section{METHOD}

\section{Subjects}

Thirty-four individuals volunteered to participate as subjects, 14 females not taking oral contraceptives, 10 females taking oral contraceptives, and 10 males. Data were not included from 5 females not taking oral contraceptives because of illness $(n=1)$, irregular menses $(n=1)$, and absence of changes in estradiol (E2), progesterone (P), and basal body temperature (BBT) characteristic of ovulating women $(n=3)$. One of the females on oral contraceptives and one male withdrew from the study. Nine subjects remained in each of the three groups. Physical histories of the participants were negative for pathologies such as head trauma or epilepsy. No subjects were taking oral or parenteral medications other than contraceptive pills where indicated. All subjects were either in college or employed and most were associated with the health professions. The ages ranged from 20 to 31 years.

\section{Procedure}

Each participant began taking her temperature 1 month prior to testing. Daily BBT was taken orally immediately after waking and before leaving bed. The BBT information was used to indicate the approximate time of ovulation for the females (Tepperman, 1973).

All subjects were tested four times. Females with ovulatory menstrual cycles were tested once at each of four selected phases of the menstrual cycle: Days 3 through 5 (during menstruation when estrogen levels are lowest), 11 through 14 (at the time of the estrogen surge immediately prior to ovulation), 18 through 21 (mid luteal when progesterone levels are highest), and 26 through 28 (premenstrually). The phase in which the first testing was done was counterbalanced across subjects. Females on oral contraceptives were off the pill 7 days and on 21 days. These women were tested 1 to 2 days prior to resuming the pill and at approximately 7-day intervals while taking the pill. The first testing was counterbalanced so that each subject began her testing sequence at various stages of the imposed cycle. Males were tested at 7-day intervals. For purposes of control and data analysis, a male's lowest temperature was considered Phase 2 and subsequent phases were assigned sequentially.

At each testing interval, data from nine behavioral and selfreport measures which were part of a larger investigation of the relationship of hormones to mood and behavior were collected. Three simple repetitive tasks and two tasks requiring inhibition were relevant to the present report. The simple repetitive tasks were: (1) the color-reading and (2) the color-naming portions of the Stroop (Stroop, 1935) and (3) the Digit Symbol subtest of the Wechsler Adult Intelligence Scale (Wechsler, 1955). Note that neither of the two portions of the Stroop is the interference task. The tasks requiring inhibition were: (1) the Embedded Figures Test (EFT) (Witkin, 1950) and (2) a subtraction task (ST) (Broverman et al., 1968) requiring subjects to count backward in steps of three and seven. The EFT involves finding an abstract geometric figure in an embedded context. To be successful in identifying the figure, one must be able to inhibit attention to the overall organization in favor of less salient features. To count backward, one must be able to inhibit the overlearned tendency to count forward. These tests are representative of the measures used by Broverman and his associates.

Serum from blood samples taken by venipuncture at each of the four testing sessions was analyzed by radioimmunoassay for E2, $P$, testosterone $(T)$, follicle stimulating hormone, and luteinizing hormone. Plasma steroids (estradiol and progesterone) were measured by specific radioimmunoassay techniques, as previously published (Mishell, Nakamura, Crosignani, Stone, Kharma, Nagato, \& Thorneycroft, 1971; Thorneycroft \& Stone, 1972). Briefly, $1 \mathrm{ml}$ of plasma is extracted twice with two volumes of $5 \mathrm{cc}$ anhydrous ether and dried under nitrogen. To estimate the percent recovery, $500 \mathrm{cpm}$ (counts per minute) of $3-\mathrm{H}$ progesterone or $3-\mathrm{H}$ estradiol- 
17 beta are added to the sample prior to extraction. After the extracted steroids have been dried, $0.4 \mathrm{ml}$ of assay buffer $(\mathrm{pH} \mathrm{7.2,}$ $0.1 \mathrm{M}$, phosphate buffer containing $0.9 \%$ Nace and $0.1 \%$ of unflavored gelatin) is used throughout the assay. One-tenth of a milliliter is taken to estimate recovery and $0.1 \mathrm{ml}$ (in duplicates) is incubated overnight at $4^{\circ} \mathrm{C}$, with specific anti-progesterone or anti-estradiol- 17 beta antibody, $0.1 \mathrm{ml}$ (containing 5,000 cpm) of 3-H-progesterone or 3-H-estradiol-17 beta and $0.2 \mathrm{ml}$ of assay buffer. Free-from-bound radioactive steroids are separated by adding $0.4 \mathrm{ml}$ of $0.625 \%$ dextran-coated charcoal solution in an ice bath, and by centrifugation at $4^{\circ} \mathrm{C}$ for $30 \mathrm{~min}$. The supernatant is counted in a scintillation counter (Beta Counter-Tri-Carb Packard $^{\circledR}$ ) for $10 \mathrm{~min}$ in $10 \mathrm{ml}$ of scintillation fluid solution $\left(1,000 \mathrm{ml}\right.$ of Toluene, $42 \mathrm{ml}$ of Liquifluor ${ }^{\circledR}$, and $100 \mathrm{ml}$ of BioSolve $^{\circledR}$ ). The amount of steroid present is calculated by comparing the counts per minute in each sample to those of a standard curve. The values are expressed in picograms per milliliter. ${ }^{1}$

\section{RESULTS AND DISCUSSION}

The mean serum E2 and P levels in relation to BBT are shown in Figure 1. For the females with ovulatory menstrual cycles, BBT was lowest in Phase 2, prior to the marked increase in $\mathrm{P}$, and highest in Phase 3. Progesterone was highest in Phase 3 , indicating that ovulation had occurred.

The scores for each order-of-testing position for each task were converted to $\mathrm{z}$ scores. This served to put all tests on a comparable scale as well as to score each subject's performance on a particular trial in relation to the performance of other subjects who had experienced the same number of prior testings. A method of combining test scores was developed prior to data analysis. For each phase, a subject's mean score on the two tasks requiring inhibition was subtracted from his/her mean score on the three simple repetitive tasks. This combined score is a within-subjects measure and is comparable in meaning to the measures used by Broverman and his associates to represent degree of automatization.

The prediction that females would be most automatized at the ovulatory phase (Phase 2) was confirmed. The mean automatization scores for each phase for all three groups are shown in Figure 2A. The difference between Phase 2 and the average of the other three phases for the females with ovulatory cycles was reliable $[\mathrm{F}(1,24)=7.40, \mathrm{p}=.012]$. Although the mean square error (Subjects within Groups by Phases) consists of $72 \mathrm{df}$, an error term based on only the $24 \mathrm{df}$ corresponding to the difference between Phase 2 and the average of Phases 1 , 3 , and 4 is appropriate for a test of that effect (Winer, 1971, p. 577-582). The use of a pooled error term for testing a simple effect is recommended by Winer (1971, p. 529).

Even though estrogen was somewhat higher in Phase 3 than in either Phase 1 or Phase 4, automatization would not necessarily be expected to be higher at that time. Progesterone, which was at its peak at Phase 3, generally has effects opposite to that of estrogen. For example, Holzbauer and Youdim (1973) found that progesterone administration increased MAO activity while estradiol administration reduced it. In any event, comparing Phase 2 with the average of the other three phases yields the same conclusions
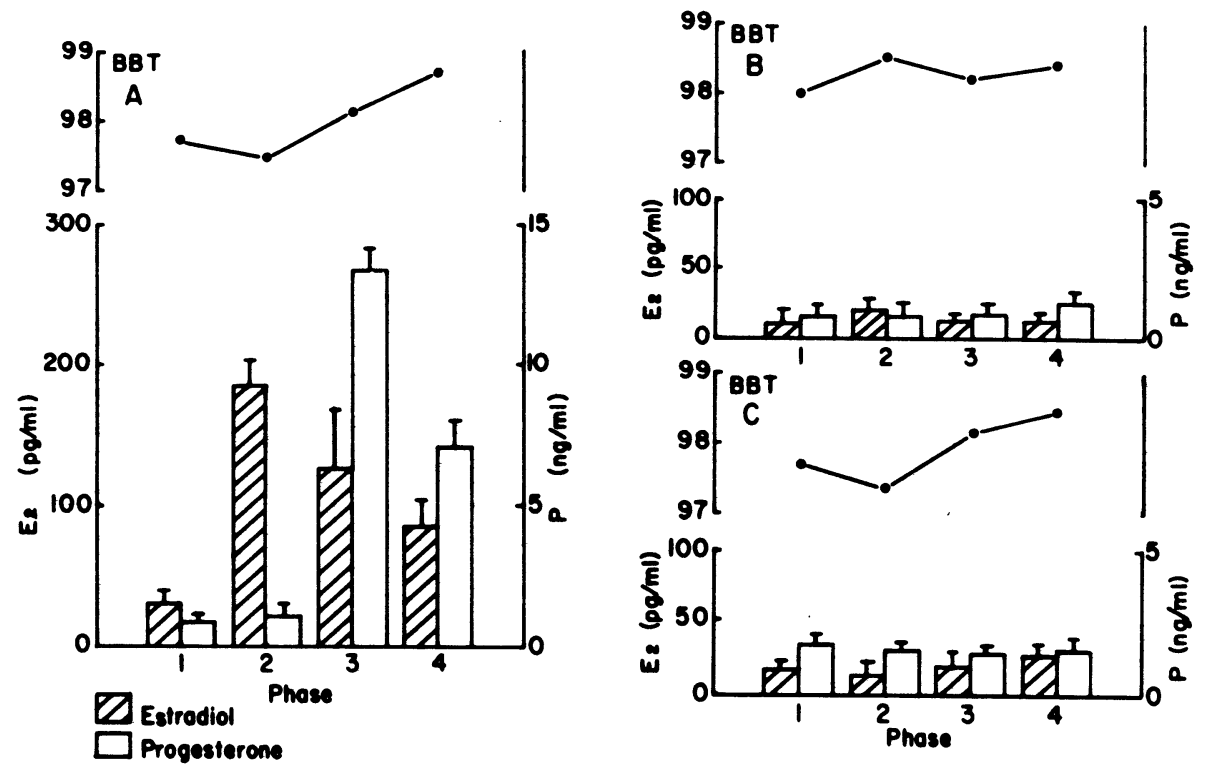

Figure 1. Plasma estradiol (E2) and progesterone (P) levels in relation to basal body temperature (BBT). BBT is represented in degrees Fahrenheit. (A) Females with ovulatory cycles. (B) Females on oral contraceptives. (C) Males. 

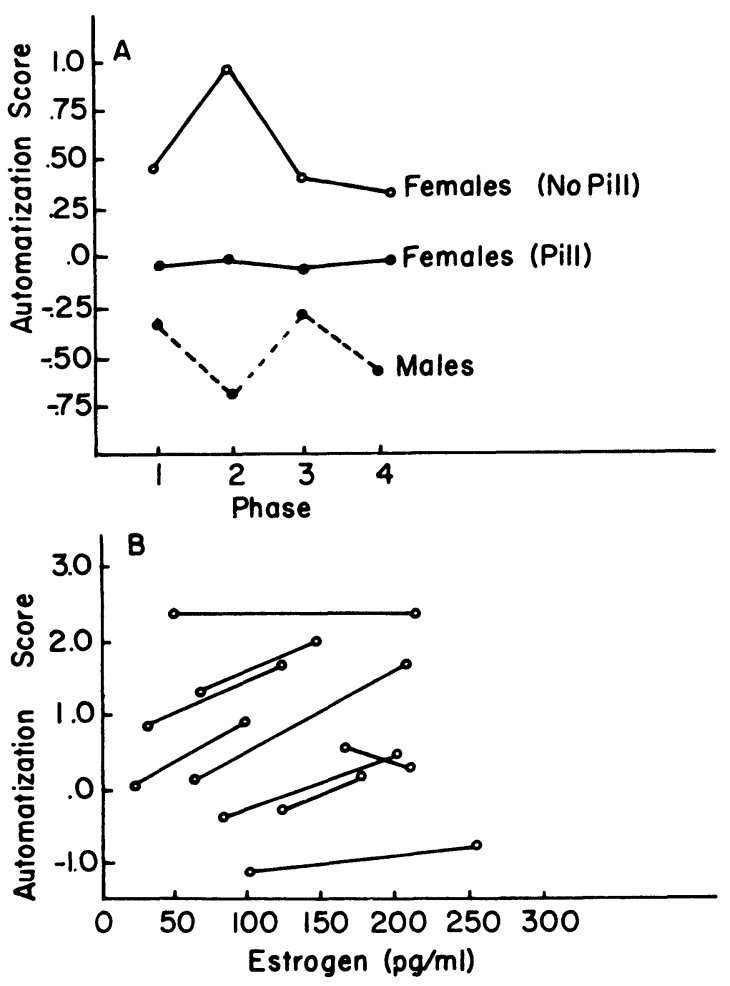

Figure 2. Mean automatization scores and hormone levels. (A) Mean automatization scores for the three groups by phase of cycle. (B) Automatization scores and $\mathrm{E} 2$ levels for each of the nine females with ovulatory cycles. For each subject, the point on the left represents the average of Phases 1,3 , and 4 , while the point on the right represents Phase 2. as would be obtained in a comparison of Phase 2 with the averages of Phase 1 and Phase 4.

Group means for each phase on the individual tests are shown in Table 1. Performance on the tasks requiring inhibition was better in Phases 1,3 , and 4 than in Phase 2 [EFT: $F(1,24)=8.35, \mathrm{p}=.008$, ST: $F(1,24)=5.81, p=.024]$. Although the speed of the color-naming test showed the predicted pattern, the best performance occurring in Phase 2, the effect was not reliable. There were virtually no differences between phases on the two other simple repetitive tasks. The effect of estrogen, therefore, appears to be primarily on the tasks requiring inhibition. This finding is consistent with the results of Waber (1976), who found the difference between early and late maturers to be significant only on the spatial tasks.

To further investigate the relationship between estrogen and automatization, the correlation between measured estradiol and automatization was computed for each female with an ovulatory cycle. The mean correlation of $.36(\mathrm{SD}=.37)$ was found to be reliable by a t test against the null hypothesis that the average population correlation $=0[\mathrm{t}(8)=2.86$, $\mathrm{p}=.021]$. Estradiol level did seem to be related to automatization in males, but the mean correlation of $.38(\mathrm{SD}=.52)$ did not reach conventional levels of significance $[\mathrm{t}(8)=2.19, \mathrm{p}=.060]$. No relationship between measured estradiol and automatization was found for the females taking oral contraceptives. This is not surprising in light of the fact that only endogenous hormones were detected by the assay.

Table 1

Group Means on Cognitive Tests Across Phases

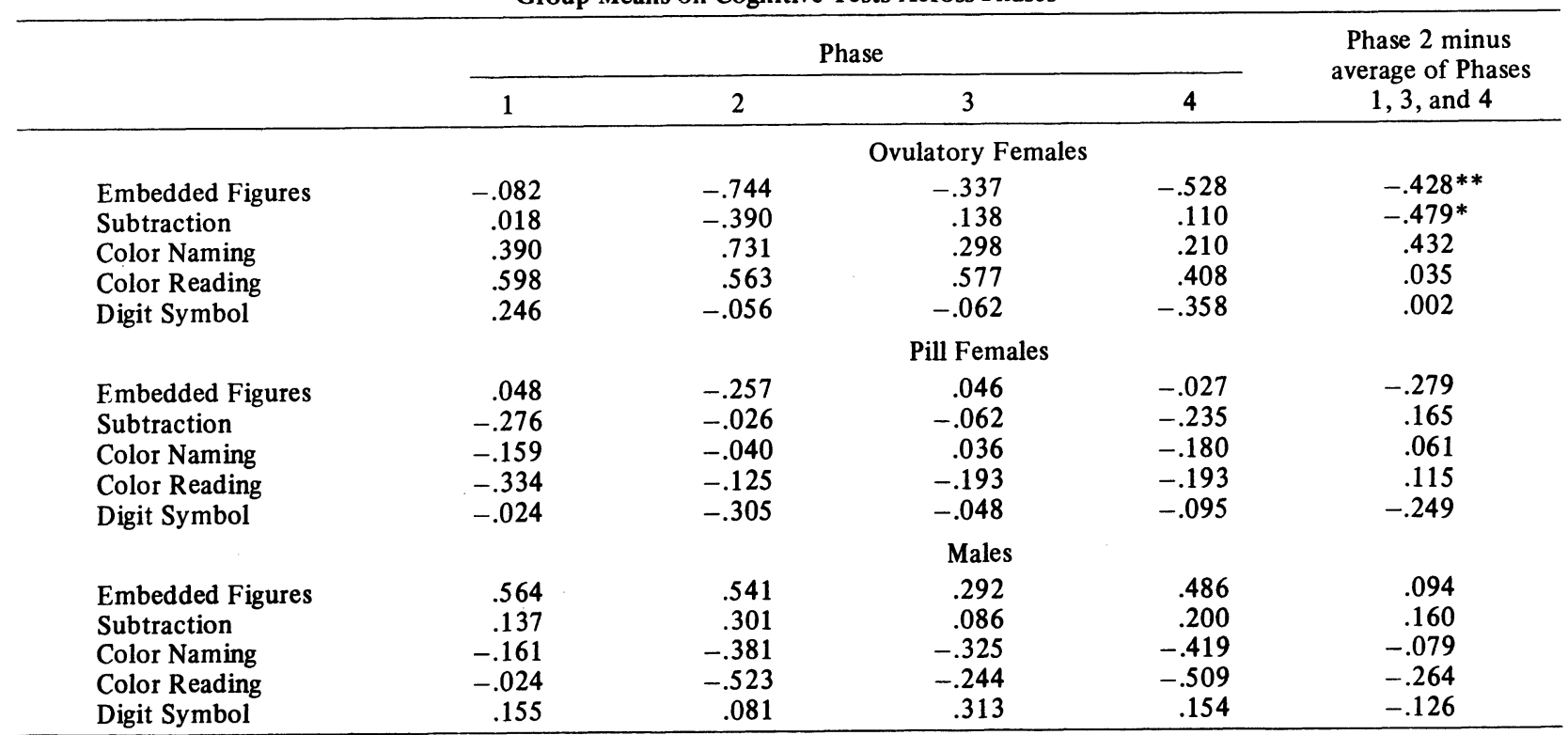

Note-All tests are scaled such that positive scores indicate better performance. Males were assigned to phase according to temperature, see text. $\quad{ }^{*} p<.025 \quad * * p<.01$ 
Progesterone and testosterone levels were analyzed in the same manner as estrogen, and no reliable effects were revealed. The correlation between the average testosterone level and the average automatization score in males was $.56, p=.12$. Given the low power with which between-subject effects were tested, this failure to find significance was not unexpected. The major concern of the study was with within-subject effects.

Figure 2B illustrates the automatization scores in Phase 2 and the average automatization score for each of the other three phases in each of the females with ovulatory cycles. The corresponding estrogen levels are also shown. Changes in automatization can be seen to be associated with changes in estrogen level. It should be pointed out that the one subject who deviates from the expected pattern was the only one whose estrogen level was higher during Phase 3 than during Phase 2. Her automatization score was also highest at Phase 3. Two additional features of Figure 2B merit comment. Although the effect of estrogen is apparent, there is no suggestion of a relationship between a women's average estrogen level and her average automatization score. Also, the within-subject effect of estrogen is small relative to between-subject differences.

As can be seen in Figure 2A, males were the least automatized and nonpill females the most automatized. Although the between-groups effect is reliable $[F(2,24)=3.91, p=.034]$, the only pairwise comparison found to be significant using the NewmanKeuls test was the difference between women with ovulatory cycles and males $[\operatorname{ts}(24)=3.95, \mathrm{p}<.05]$. Although the data suggest that women taking oral contraceptives are less automatized than other women, the difference was not significant $[\mathrm{t}(24)=$ $1.56, \mathrm{p}=.132]$. Further, because subjects were not randomly assigned to conditions, a sampling bias is possible. $^{2}$

These data provide evidence that there is a systematic fluctuation in cognitive performance over the menstrual cycle. Performance on the EFT and the ST was slightly, but consistently, lower during the ovulatory phase than during other phases of the cycle. There was a nonsignificant tendency for color naming to be fastest during the ovulatory phase. Although effects of birth control pills on performance were not clearly demonstrated, future research in which women are tested before and after beginning a program of oral contraception would be of interest.

\section{REFERENCES}

Briggs, M., \& Briggs, M. Relationship between monoamine oxidase activity and sex hormone concentration in human blood plasma. Journal of Reproductive Physiology, 1972, 24, 536-539. Broverman, D. M., Broverman, I. K., Vogel, W., Palmer, R., \& KLAIBER, E. L. Cognitive style and physical development. Child Development, 1964, 35, 1343-1359.
Broverman, D. M., \& Klaiber, E. L. Negative relationships between abilities. Psychometrika, 1969, 34, 5-20.

Broverman, D. M., Klaiber, E., Kobayashi, Y., \& Vogel, W. Roles of activation and inhibition in sex differences in cognitive abilities. Psychological Review, 1968, 75, 23-50.

CAse, R., \& Globerson, T. Field-independence and central computing space. Child Development, 1974, 45, 772-778.

Holzbauer, M., \& Youdim, M. The oestrous cycle and monoamine oxidase activity. British Journal of Pharmacology, 1973, 48, 600-608.

Klaiber, E. L., Kobay ashi, Y., Broverman, D. M., \& Hall, F. Plasma monoamine oxidase activity in regularly menstruating women and in amenorrheic women receiving cyclic treatment with estrogens and a progestin. Journal of Clinical Endocrinology, 1971, 33, 630-638.

Mishell, D., Nakamura, R., Crosignani, P., Stone, S. C., Kharma, K., Nagata, Y., \& Thorneycroft, I. Serum gonadotropin and steroid pattern during the normal menstrual cycle. American Journal of Obstetrics and Gynecology, 1971, $111,60-65$.

Pascual-Leone, J. A mathematical model for the transition rule in Piaget's developmental stages. Acta Psychologica, 1970, 63, 301-345.

Petersen, A. C. Physical androgyny and cognitive functioning in adolescence. Developmental Psychology, 1976, 12, 524-533.

Redmond, D. E., MurPhy, D. L., Baulu, J., Ziegler, M. G., \& LAKE, C. R. Menstrual cycle and ovarian hormone effects on plasma and platelet monoamine oxidase (MAO) and plasma dopamine-beta-hydroxylase (DBH) activities in the rhesus monkey. Psychosomatic Medicine, 1975, 37, 417-428.

Robinson, D. S., Sourkes, T. L., Nies, A., Harris, L. S., Spector, S., Bartlett, D. L., \& KaYe, I. S. Monoamine metabolism in human brain. Archives of General Psychiatry, 1977, 34, 89-92.

Sherman, J. A. Problem of sex differences in space perception and aspects of intellectual functioning. Psychological Review, 1967, 74, 290-300.

SмIтн, I. M. Spatial ability: Its educational and social significance. London: University of London Press, 1964.

STROop, J. R. Studies of interference in serial verbal reactions. Journal of Experimental Psychology, 1935, 18, 643-660.

Tepperman, J. Metabolic and endocrine physiology. Chicago: Yearbook Medical, 1973.

Thorneycroft, I., \& Stone, S. C. Radioimmunoassay of serum progesterone in women receiving oral contraceptive steroids. Contraception, 1972, 5, 129-146.

WABER, D. P. Sex differences in cognition: A function of maturation rate? Science, 1976, 192, 572-574.

WABER, D. P. Sex differences in mental abilities, hemispheric lateralization, and rate of physical growth at adolescence. Developmental Psychology, 1977, 13, 29-38.

WECHSLER, D. Manual for the Wechsler Adult Intelligence Scale. New York: The Psychological Corporation, 1955.

WINER, B. J. Statistical principles in experimental design. New York: McGraw-Hill, 1971.

Witkin, H. A. Individual differences in ease of perception of embedded figures. Journal of Personality, 1950, 19, 1-15.

YANG, H., \& NEFF, N. H. The monoamine oxidases of brain: Selective inhibition with drugs and the consequences for the metabolism of the biogenic amines. Journal of Pharmacological and Experimental Therapeutics, 1974, 189, 733-740.

Zolovick, A. J., Pearse, R., Boehlke, K. W., \& Eleftheriou, B. E. Monoamine oxidase activity in various parts of the rat brain during the estrus cycle. Science, 1966, 154, 649.

\section{NOTES}

1. Specific anti-progesterone antibody was kindly donated by Dr. Dan Tulchinsky, Department of Ob-Gyn, Harvard School of Medicine. Specific anti-estradiol-17 beta antibody was kindly 
donated by Dr. Uwe Goebelsmann, Department of Ob-Gyn, University of Southern California School of Medicine.

2. Subjects on oral contraceptives were taking various forms of the combination pill which, depending on the specific pill, could contain from 50 to $100 \mathrm{mcg}$ of Estrogen and .5 to $2.0 \mathrm{mg}$ of Progestin. Included among the pills were Ovral; Orthonovum 2,
$1+50$ and $1+80$; Norinyl, $1+50$ and $1+80$; and Demulen. These women did not appear to differ systematically in age or sociocultural background from other women in the study.

(Received for publication April 14, 1977; revision accepted October 27, 1977.) 